\title{
BOUNDS ON THE SPECTRAL RADIUS OF \\ HADAMARD PRODUCTS OF POSITIVE \\ OPERATORS ON $\ell_{P}$-SPACES*
}

\author{
ANTON R. SCHEP ${ }^{\dagger}$
}

\begin{abstract}
Recently, K.M.R. Audenaert (2010), and R.A. Horn and F. Zhang (2010) proved inequalities between the spectral radius of Hadamard products of finite nonnegative matrices and the spectral radius of their ordinary matrix product. We will prove these inequalities in such a way that they extend to infinite nonnegative matrices $A$ and $B$ that define bounded operators on the classical sequence spaces $\ell_{p}$.
\end{abstract}

Key words. Hadamard product, Spectral radius, Nonnegative matrix, Positive operator, Matrix inequality.

AMS subject classifications. 15A45, 15B48, 47B65.

1. Introduction. In [1], K.M.R. Audenaert proved a conjecture of X. Zhan [5] by proving that for nonnegative $n \times n$ matrices $A$ and $B$ the spectral radius $\rho(A \circ B)$ of the Hadamard product satisfies

$$
\rho(A \circ B) \leq \rho^{\frac{1}{2}}((A \circ A)(B \circ B)) \leq \rho(A B),
$$

where $A B$ denotes the ordinary matrix product of $A$ and $B$. These inequalities were established via an intricate inequality involving traces. Using the fact that the Hadamard product is a principal submatrix of the Kronecker product $A \otimes B$, R.A. Horn and F. Zhang [3] proved that

$$
\rho(A \circ B) \leq \rho^{\frac{1}{2}}(A B \circ B A) \leq \rho(A B) .
$$

They noted in their paper that their methods could be used to derive the right-hand side of the inequalities of Audenaert, but that the left-hand side appeared to be deeper.

In this paper, we will show that we can derive the left-hand side of this inequality without using the characterization of the spectral radius as a limit of traces. We will prove in fact the inequalities

$$
\rho(A \circ B) \leq \rho^{\frac{1}{2}}((A \circ A)(B \circ B)) \leq \rho^{\frac{1}{2}}(A B \circ B A) \leq \rho(A B)
$$

* Received by the editors on November 15, 2010. Accepted for publication on April 16, 2011. Handling Editor: Roger A. Horn.

${ }^{\dagger}$ Department of Mathematics, University of South Carolina, Columbia, SC 29212, USA (schep@math.sc.edu). 
in such a way that the proofs are valid for positive operators on $\ell_{p}$ spaces, where we will assume throughout the paper that $1 \leq p<\infty$. In this context, the method of Audenaert is not applicable, while the method of Horn and Zhang is not directly applicable, but we do follow many of the ideas of [3]. Our main tool is the formula $\rho(A)=\lim _{n \rightarrow \infty}\left\|A^{n}\right\|^{\frac{1}{n}}$, where $\|A\|$ denotes the operator norm of $A$ on $\ell_{p}$, and $\ell_{p}$ is equipped with the usual $\|\cdot\|_{p}$-norm.

2. Hadamard product of matrices of operators on $\ell_{p}$. First we recall some terminology and notations. We write $x \geq 0$ for $x=\left(\xi_{n}\right) \in \ell_{p}$, whenever $\xi_{n} \geq 0$ for all $n \geq 1$, and we denote by $\ell_{p}^{+}$the set of all $x \geq 0$ in $\ell_{p}$. A bounded linear operator $A: \ell_{p} \rightarrow \ell_{p}$ is called positive (denoted by $A \geq 0$ ) if $A x \geq 0$ for all $x \in \ell_{p}^{+}$. As we assume $p<\infty$, every bounded operator on $\ell_{p}$ has a matrix representation with respect to the standard basis, and we will identify the operator with its matrix. In case $A \geq 0$, we have $A=\left[a_{i j}\right]$, where each $a_{i j} \geq 0$. We will use frequently that if $0 \leq A \leq B$ on $\ell_{p}^{+}$(i.e., $A-B \geq 0$ ), then $\|A\| \leq\|B\|$. Given two bounded linear operators $A=\left[a_{i j}\right], B=\left[b_{i j}\right]$ on $\ell_{p}$, we define $A \circ B$ by the matrix $\left[a_{i j} b_{i j}\right]$.

Our first lemma shows that $A \circ B$ defines a bounded linear operator on $\ell_{p}$. This result is known in greater generality than stated here, see e.g. [2], but for completeness we include a short proof .

Lemma 2.1. Let $A$ and $B$ be positive linear operators on $\ell_{p}$. Then $A \circ B$ is a positive linear operator on $\ell_{p}$ and $\|A \circ B\| \leq\|A\|\|B\|$.

Proof. By homogeneity it is sufficient to prove that $\|A \circ B\| \leq 1$, whenever $\|A\|=\|B\|=1$. Assume $B=\left[b_{i j}\right]$. From $\|B\|=1$ it follows that $b_{i, j} \leq 1$ for all $i, j$, so that $0 \leq A \circ B \leq A$. This implies immediately that $A \circ B$ is a positive operator from $\ell_{p}$ to $\ell_{p}$ and $\|A \circ B\| \leq 1$.

We denote by $A^{\left(\frac{1}{2}\right)}$ the pointwise ("Hadamard") square root of the operator $A$. The following lemma is a special case of the Krivine calculus in Banach lattices; see Proposition 1.d.2 of [4].

Lemma 2.2. Let $A$ and $B$ be positive operators on $\ell_{p}$. Then $A^{\left(\frac{1}{2}\right)} \circ B^{\left(\frac{1}{2}\right)}$ is a positive operator on $\ell_{p}$ and $\left\|A^{\left(\frac{1}{2}\right)} \circ B^{\left(\frac{1}{2}\right)}\right\| \leq\|A\|^{\frac{1}{2}}\|B\|^{\frac{1}{2}}$.

Proof. We use the identity $(a b)^{\frac{1}{2}}=\min \left\{\frac{t^{2}}{2} a+\frac{1}{2 t^{2}} b ; t>0\right\}$ to get

$$
A^{\left(\frac{1}{2}\right)} \circ B^{\left(\frac{1}{2}\right)} \leq \frac{t^{2}}{2} A+\frac{1}{2 t^{2}} B \text { for all } t>0 .
$$

This implies that $A^{\left(\frac{1}{2}\right)} \circ B^{\left(\frac{1}{2}\right)}$ is a positive operator on $\ell_{p}$, and

$$
\left\|A^{\left(\frac{1}{2}\right)} \circ B^{\left(\frac{1}{2}\right)}\right\| \leq \frac{t^{2}}{2}\|A\|+\frac{1}{2 t^{2}}\|B\| \text { for all } t>0
$$

By taking the minimum over $t$, we get the desired inequality. 
A more general version of the following proposition for integral operators appears in [2]. As it is an essential step of the main result, we include a simple proof.

Proposition 2.3. Let $A, B, C$ and $D$ be positive operators on $\ell_{p}$. Then we have $(A \circ B)(C \circ D) \leq((A \circ A)(C \circ C))^{\left(\frac{1}{2}\right)} \circ((B \circ B)(D \circ D))^{\left(\frac{1}{2}\right)}$.

Proof. Let $\left[a_{i, j}\right],\left[b_{i, j}\right],\left[c_{i, j}\right]$, and $\left[d_{i, j}\right]$ denote the matrices of the operators $A, B$, $C$, and $D$ respectively. Then the matrix of the operator product $(A \circ B)(C \circ D)$ is given by $\left[\sum_{l=1}^{\infty} a_{i l} b_{i l} c_{l j} d_{l j}\right]$. From the Cauchy-Schwarz inequality we get

$$
\sum_{l=1}^{\infty} a_{i l} b_{i l} c_{l j} d_{l j} \leq\left(\sum_{l=1}^{\infty} a_{i l}^{2} c_{l j}^{2}\right)^{\frac{1}{2}}\left(\sum_{l=1}^{\infty} b_{i l}^{2} d_{l j}^{2}\right)^{\frac{1}{2}}
$$

which is the entrywise statement of the assertion.

Corollary 2.4. Let $A$ and $B$ be positive operators on $\ell_{p}$. Then we have

$$
(A \circ B)^{2} \leq((A \circ A)(B \circ B))^{\left(\frac{1}{2}\right)} \circ((B \circ B)(A \circ A))^{\left(\frac{1}{2}\right)}
$$

and

$$
\left(A^{\left(\frac{1}{2}\right)} \circ B^{\left(\frac{1}{2}\right)}\right)^{2} \leq\left(A^{2}\right)^{\left(\frac{1}{2}\right)} \circ\left(B^{2}\right)^{\left(\frac{1}{2}\right)} .
$$

Proof. For the first inequality, we take $D=A$ and $C=B$ in Proposition 2.3. For the second inequality, we substitute $A^{\left(\frac{1}{2}\right)}$ for both $A$ and $C$, and $B^{\left(\frac{1}{2}\right)}$ for both $B$ and $D$ in Proposition 2.3.

One final pointwise inequality we need is the following inequality, which is proved in [3] for finite matrices by means of tensor product arguments.

Lemma 2.5. Let $A$ and $B$ be positive operators on $\ell_{p}$. Then we have

$$
(A \circ A)(B \circ B) \leq A B \circ A B .
$$

Proof. Let $\left[a_{i, j}\right]$ and $\left[b_{i, j}\right]$ denote the matrices of $A$ and $B$, respectively. Then the $(i, j)$ th entry of $(A \circ A)(B \circ B)$ is $\sum_{k=1}^{\infty} a_{i k}^{2} b_{k j}^{2}$, and

$$
\sum_{k=1}^{\infty} a_{i k}^{2} b_{k j}^{2} \leq\left(\sum_{k=1}^{\infty} a_{i k} b_{k j}\right)^{2}
$$

which proves the inequality.

TheOREM 2.6. Let $A$ and $B$ be positive operators on $\ell_{p}$. Then we have

$$
\rho\left(A^{\left(\frac{1}{2}\right)} \circ B^{\left(\frac{1}{2}\right)}\right) \leq \rho(A)^{\frac{1}{2}} \rho(B)^{\frac{1}{2}} \text { and } \rho(A \circ B) \leq \rho(A) \rho(B) .
$$


Proof. The first inequality is a special case of a theorem in [2]. For completeness we include a proof. From equation (2.2) it follows that

$$
\left(A^{\left(\frac{1}{2}\right)} \circ B^{\left(\frac{1}{2}\right)}\right)^{2^{n}} \leq\left(A^{2^{n}}\right)^{\left(\frac{1}{2}\right)} \circ\left(B^{2^{n}}\right)^{\left(\frac{1}{2}\right)} .
$$

Taking norms we get

$$
\left\|\left(A^{\left(\frac{1}{2}\right)} \circ B^{\left(\frac{1}{2}\right)}\right)^{2^{n}}\right\| \leq\left\|A^{2^{n}}\right\|^{\frac{1}{2}}\left\|B^{2^{n}}\right\|^{\frac{1}{2}}
$$

Taking $2^{n}$ th roots on both sides, and then the limit for $n \rightarrow \infty$, we get

$$
\rho\left(A^{\left(\frac{1}{2}\right)} \circ B^{\left(\frac{1}{2}\right)}\right) \leq \rho(A)^{\frac{1}{2}} \rho(B)^{\frac{1}{2}} .
$$

To get the second inequality, we first prove that $\rho(A \circ A) \leq \rho(A)^{2}$. To this end, take $A=B$ in the preceding lemma to get $(A \circ A)^{2} \leq A^{2} \circ A^{2}$. Iterating this we get $(A \circ A)^{2^{n}} \leq A^{2^{n}} \circ A^{2^{n}}$. Taking norms and using Lemma 2.1, we get

$$
\left\|(A \circ A)^{2^{n}}\right\| \leq\left\|A^{2^{n}}\right\|^{2}
$$

which implies as before that $\rho(A \circ A) \leq \rho(A)^{2}$. To obtain the second inequality, use the first inequality to get

$$
\rho(A \circ B) \leq \rho(A \circ A)^{\frac{1}{2}} \rho(B \circ B)^{\frac{1}{2}},
$$

from which the second inequality follows, since $\rho(A \circ A) \leq \rho(A)^{2}$ and $\rho(B \circ B) \leq$ $\rho(B)^{2}$.

We are now in a position to prove the main inequalities.

Theorem 2.7. Let $A$ and $B$ be positive operators on $\ell_{p}$. Then we have

$$
\rho(A \circ B) \leq \rho^{\frac{1}{2}}((A \circ A)(B \circ B)) \leq \rho^{\frac{1}{2}}(A B \circ B A) \leq \rho(A B),
$$

and

$$
\rho\left(A^{\left(\frac{1}{2}\right)} \circ B^{\left(\frac{1}{2}\right)}\right) \leq \rho(A B)^{\frac{1}{2}} .
$$

Proof. From (2.1) we get

$$
(A \circ B)^{2} \leq((A \circ A)(B \circ B))^{\left(\frac{1}{2}\right)} \circ((B \circ B)(A \circ A))^{\left(\frac{1}{2}\right)} .
$$

Applying (2.2) to powers on the right hand side, we get

$$
(A \circ B)^{2^{n}} \leq\left(((A \circ A)(B \circ B))^{2^{n-1}}\right)^{\left(\frac{1}{2}\right)} \circ\left(((B \circ B)(A \circ A))^{2^{n-1}}\right)^{\left(\frac{1}{2}\right)} .
$$


Taking norms, we get

$$
\left\|(A \circ B)^{2^{n}}\right\| \leq\left\|((A \circ A)(B \circ B))^{2^{n-1}}\right\|^{\frac{1}{2}}\left\|((B \circ B)(A \circ A))^{2^{n-1}}\right\|^{\frac{1}{2}} .
$$

Now taking $2^{n}$ th roots and limits, we get

$$
\rho(A \circ B) \leq \rho((A \circ A)(B \circ B))^{\frac{1}{4}} \rho((B \circ B)(A \circ A))^{\frac{1}{4}}=\rho((A \circ A)(B \circ B))^{\frac{1}{2}} .
$$

From $(A \circ A)(B \circ B) \leq A B \circ A B$ we now conclude that

$$
\rho((A \circ A)(B \circ B)) \leq \rho(A B \circ A B) \leq \rho(A B)^{2},
$$

which completes the proof of (2.3). To prove the inequality (2.4), we just substitute $A^{\left(\frac{1}{2}\right)}$ for $A$, and $B^{\left(\frac{1}{2}\right)}$ for $B$ in the left-most inequality of (2.3).

One may ask whether it is necessary to restrict oneself to $\ell_{p}$-spaces. The answer is no. All of our results are valid for positive operators on more general sequence spaces. On the other hand, we cannot extend the main result to function spaces. The reason is that the Hadamard product for positive operators on function spaces on non-atomic measure spaces does not in general define a bounded operator, e.g., if $A$ denotes the integral operator on $L_{2}[0,1]$ with kernel $\frac{1}{\sqrt{|x-y|}}$, then the kernel $\frac{1}{|x-y|}$ of $A \circ A$ does not define an operator on $L_{2}[0,1]$, or any other $L^{p}$ space. On the other hand, as already noted in [2], if $A$ and $B$ are positive integral operators on a Banach function space $L_{p}$, then $A^{\left(\frac{1}{2}\right)} \circ B^{\left(\frac{1}{2}\right)}$ defines a bounded operator on $L_{p}$. We have therefore the following result, which can be proved along the exact same lines as the main result Theorem 2.7.

TheOrem 2.8. Let $A$ and $B$ be positive integral operators on $L_{p}$. Then we have

$$
\rho\left(A^{\left(\frac{1}{2}\right)} \circ B^{\left(\frac{1}{2}\right)}\right) \leq \rho(A B)^{\frac{1}{2}} .
$$

\section{REFERENCES}

[1] K.M.R. Audenaert. Spectral radius of Hadamard product versus conventional product for nonnegative matrices. Linear Algebra Appl., 432(1):366-368, 2010.

[2] R. Drnovšek and A. Peperko. Inequalities for the Hadamard weighted geometric mean of positive kernel operators on Banach function spaces. Positivity, 10:613-626, 2006.

[3] R.A. Horn and F. Zhang. Bounds on the spectral radius of a Hadamard product of nonnegative or positive semidefinite matrices. Electron. J. Linear Algebra, 20:90-94, 2010.

[4] J. Lindenstrauss and L. Tzafriri. Classical Banach Spaces II. Ergebnisse der Mathematik und ihrer Grenzgebiete [Results in Mathematics and Related Areas], 97, Springer-Verlag, Berlin, 1979.

[5] X. Zhan. Unsolved matrix problems. Talk given at Advanced Workshop on Trends and Developments in Linear Algebra, ICTP, Trieste, Italy, July 6-10, 2009. 